\title{
Project Control and Computational Intelligence: Trends and Challenges
}

\author{
José Alejandro Lugo García ${ }^{* 1}$, Anié Bermudez Peña ${ }^{1}$, Pedro Yobanis Piñero Pérez ${ }^{\mathbf{1}}$ \\ ${ }^{1}$ Laboratory of Project Management Research, University of Information Sciences, Carretera a San Antonio de los \\ Baños, km 2 1⁄2, Boyeros, La Habana, ZIP Code: 19370, Cuba \\ E-mail: jalugogarcia@gmail.com,abp@uci.cu,ppp@uci.cu; www.uci.cu \\ Rafael Bello Pérez \\ Department of Computer Science, Central University "Martha Abreu” of Las Villas, Santa Clara, Cuba \\ E-mail: rbellop@uclv.edu.cu; www.uclv.edu.cu
}

Received 12 September 2015

Accepted 30 October 2016

\begin{abstract}
Project monitoring and control by using key performance indicators has become a widespread method for decisionmaking in project-oriented organizations. However, the current schools and IT tools created for this purpose require an upgrade in design due to imprecision, vagueness or uncertainty present in the raw data and changing conditions in management styles. Moreover, the use of proprietary technologies in developing nations represents high costs for governments and obstacles to achieving its technological sovereignty. This paper studies the trends and challenges in project control through computational intelligence methods. It also examines schools and technological tools to manage projects, as well as open source software for the application of computational intelligence techniques over the past decades. Current tendencies and improvement areas, valuing niche markets with high applicability around the thematic goal it is also analyzed. The contribution of this study is related to the predicted necessity of constructing new models and IT tools for project control which integrate machine learning-based approaches and treatment of imprecision, vagueness or uncertainty in the information, using key performance indicators linked to fundamental knowledge areas. The implementation of new libraries for learning evaluation in project control with open source software tools, opens a field of research related to increase technological integration with IT project management tools. The content under discussion provides support to improve decision-making in project-oriented organizations.
\end{abstract}

Keywords: Computational Intelligence, decision-making, open source software, project control.

\section{Introduction}

Management problems require solution methods and tools appropriate to their complexity. Actions to project control arise from analyzing information resulting from the particular activities during their management, through the assessment of key indicators obtained from the collection of performance data during execution, facilitating decision-making [1].

According to current standards and models agreed [1] [2], the most used key performance indicators
(KPIs) for project control are linked to knowledge areas related to managing time (scheduling), cost (budgeting) and quality. Additionally, investigations such as those conducted by The Standish Group in 2013 [3] and Pacelli [4], identify human resources and logistics (material resources) respectively, as a key factor to consider for succeeding in projects. Then, it is also important to include these areas as fundamental knowledge areas and its respective monitoring indicators to conduct such control. Therefore, the main topics in project control are related to monitoring the

*Corresponding author. 
status of the derivate information from the knowledge areas of Time, Cost, Quality, Logistics and Human Resources management ${ }^{\mathrm{a}}$. After an analysis of each area is performed, a general evaluation of the project is required in order to further decision-making steps.

Project control is related to the management of numerical and linguistic data, noise caused by measurement errors, appreciation of people and vague concepts for decision-making. The insufficiencies in the management of these data and incorrect evaluation of projects, lead to many economic losses with high social impact. Among the main causes of failure in this area can be cited [5] [6] [7]:

- Lacks in knowledge of good practices.

- The limited experience in the control and monitoring of projects.

- Deficits in automated calculation of indicators based on evidence taken as a result of the product development process, covering key knowledge areas of project management.

- Weaknesses in the information technology (IT) tools for automatic or semi-automatic evaluation of projects.

- Difficulties in treating imprecision, vagueness or uncertainty contained in the information.

- Deficiencies in learning and adapting the evaluation of the project execution given the level of maturity reached by the organization during its continuous improvement.

- Use of proprietary technologies to support project management in developing countries represents high costs for governments and obstacles to achieving its technological sovereignty [8].

The need of considering imprecision, vagueness or uncertainty, as well as learning and adaptation in the processing of information for project control, has led to the development of various alternative solutions that claim to be robust [9]. One alternative is the introduction of computational intelligence techniques [10], within which soft computing techniques are included [11].

In the last decade, an increasing field of research has proposed useful solutions for project control based on computational intelligence approaches. However, projected results have methodological deficiencies and

\footnotetext{
${ }^{a}$ Other knowledge areas like Scope, Risks or Integration can also be monitored, but it is going to consider the previous five mentioned as the most important ones, to establish a general project evaluation status.
}

gaps related to integration with IT project management tools that hinder the usability and applicability of the obtained models.

In this paper, we investigate the trends and challenges in project control through computational intelligence methods. We also examine schools and technological tools to manage projects, as well as open source software for the application of computational intelligence techniques over the past decades. Current tendencies and improvement areas, valuing niche markets with high applicability around the thematic goal it is also analyzed. Finally, conclusions and recommendations for future work are presented.

\section{Project Management: Schools and IT tools}

Project management schools are known as: more representative best practices, methodologies and / or existing standards at international level, which proposes activities, techniques, and tools for such purposes.

\subsection{Project Management Body of Knowledge (PMBOK)}

Developed by the Project Management Institute PMBOK in its fifth edition [1] provides guidelines for the project life cycle and related processes. It is one of the most recognized internationally management projects standards. It is composed of five basic process groups: Initiating, Planning, Executing, Monitoring and Controlling and Closing. These groups have to turn to internal processes which establish a methodology to be followed by project managers. PMBOK proposes ten areas of knowledge, namely: Integration Management, Scope Management, Time Management, Cost Management, Quality Management, Human Resource Management, Communications Management, Risk Management, Procurement Management and Stakeholder Management.

PMBOK proposes to monitor the execution of the project during all its life cycle to provide constructive feedback, discover unknown or unresolved issues and encourage continuous improvement to future periods. For this, it suggests the use of indicators. However, the proposed indicators are mainly related to knowledge areas of scope, cost, time and quality, leaving aside other important areas such as human resource management and logistics. On the evaluation, focuses its attention to performance area of human resources 
and the project in general, directing their procedure to verbal actions generically established, becoming very difficult in application to real and specific project management environments.

PMBOK recognizes the importance of evaluation, treatment of uncertainty in information and influence of IT tools in the success of the project. However, does not propose specific mechanisms to implement such shortterm purposes. It is, therefore, the responsibility of organizations to achieve its correct implementation.

\subsection{Projects in Controlled Environments (PRINCE2)}

PRINCE2 provides a set of best practices around project management, which covers the control, administration, and organization of projects [12]. Proposes using indicators such as Return of Investment of the project (ROI), Cost and Increased Risks associated with the project, pointing to good practice action compile, store them for future use in the processes of Project Start. It also proposes to implement any kind of metrics that can be useful in assessing whether the product meets the requirements as agreed with the client. PRINCE2 defines six areas related tolerance Time, Cost, Scope, Risk, Quality and Benefit which aims to report and assess the progress of the project through graphical planned performance vs. actual performance milestones and earned value management.

However, PRINCE2 does not propose concrete methods for learning and adaptation in project evaluation given the level of maturity reached by the organization during its continuous improvement. Nor it refers to the treatment of imprecision, vagueness or uncertainty contained in the information that is managed.

\subsection{ISO 21500: 2012}

ISO 21500: 2012 [2] provides a guide for direction and project management. Identify processes and management (Home, Planning, Implementation, Control, Closure) grouped by subject groups (Integration Party concerned, Scope, Resource, Time, Cost, Risk, Quality, Acquisition, Communication) capable of distributing and managing the relevant project information setting inputs and outputs. It can be used as a link between different business processes and project management. It complements the ISO 10006 standard for quality management, especially in the area of continuous improvement making enhancement processes needed in operations with minimal disruption to the production and services.

The process of collecting lessons learned focuses on knowledge management. It includes a paragraph in the closing process (subgroup Integration) on the collection of the experience gained in the decision-oriented preventive measures in project organization. This allows the project team and stakeholders, face challenging situations proactively to achieve better performance. It is recognized that the management of communication enriches lessons learned, creating a common understanding of the project and a shared vision for a successful teamwork. However, as happens with schools discussed above, it does not propose concrete mechanisms to ensure the applicability of its principles in the short term. On the other hand, it does not suggest techniques for learning and adaptation of project evaluation given the level of maturity reached by the organization for continuous improvement. It does not address the treatment of the imprecision, vagueness or uncertainty contained in the information.

\subsection{Capability Maturity Model Integration (CMMI)}

Capability Maturity Model Integration (CMMI) is developed and maintained by the Software Engineering Institute [13]. It is a model for improving and assessing software development processes. CMMI proposes five maturity levels: 1-Initial, 2-Administered, 3-Defined, 4Administered quantitatively and 5-Optimized; for a total of 16 key process areas spread over 5 levels.

CMMI recognizes that assessments of process and product must be obtained from the analysis and interpretation of metrics (of which are derivable indicators) defined by the organization. The areas where the use of metrics and indicators are more reflected to controlling the development processes are: Measurement and Analysis, Monitoring and Control Project, Project Planning, Quality Assurance Process and Product, Integrated Project Management and Quantitative Management of the Project. However, the model focuses on the verbal description of the processes and does not define specific indicators or concrete methods for learning and adaptation in the evaluation of projects according to the level of maturity reached by the organization.

The model addresses continuous improvement through the optimization of processes within the organization, but only when it reaches maturity level 5 . 
To achieve this, it states that projects must periodically update the definition of their processes and implement mechanisms to evaluate the effectiveness of the ongoing implementation regarding the performance targets set by the organization, project or individual. However, in the judgment of the authors, continuous improvement and management must be present at all times, not just when the organization reaches its maximum level of maturity. Moreover, CMMI recognizes the importance of using tools such as information systems to support decisionmaking. However, it leaves the organization responsible for implementing them, not referring to the treatment of imprecision, vagueness or uncertainty contained in the information that is managed.

\subsection{Project control and IT tools}

Given the steady increase in complexity to manage information related to project control, it is essential to use tools that support it. In the last decades have been developed a large number of these tools, also known as Project Management Information Systems (PMIS).

In July 2016, the authors performed a comparative study to 121 of the most representative IT tools to support project management worldwide. The study was based on the tools shown on the reports of the Gartner company [14] [15], and others found in social networks. The study included tools based on proprietary and open source software (see Appendix A). The results showed that only eight tools based on open source software implement indicators reports and data analysis features. Moreover, it became apparent that none of these tools performed treatment of imprecision, vagueness or uncertainty in the evaluation of projects. The imprecision contained in these tools is evident at the moment when users insert numerical values on fields like "actual percentage of execution completion", "estimated" or "actual time spent on tasks". Vagueness is present during assignment or use of linguistic terms to identify features such as "priority" or "complexity" of the tasks. Uncertainty meanwhile, appears at the moment when the heads of tasks and projects assigned evaluations, which logically depend on human perception and the experience of the evaluator. Neither exists evidence in the analyzed tools that demonstrate the implementation of features related to learning and adaptation in the evaluation of projects, according to the level of maturity reached by the organization during its continuous improvement.

While many of these tools satisfy the needs of some customers, they not always cover everyone's expectations because specific or combined topics related to price, license, support or inadequacies in the handling of certain data. Furthermore, the use of wrong technology it is observed as one of the big mistakes during project management. In this sense, Pacelli [4] points out that the choice of a technology should depend on the environment in which the organization operates. Also, you can choose the right technology and personnel not have the necessary skills to handle it. Therefore, it is also important to define a methodological strategy to implement appropriate technology to use in each case.

\section{Computational Intelligence. Components and Technological Tools}

In the context of computer science, Computational Intelligence (CI) is part of a field where Artificial Intelligence and Soft Computing converges [10]. See Figure 1:

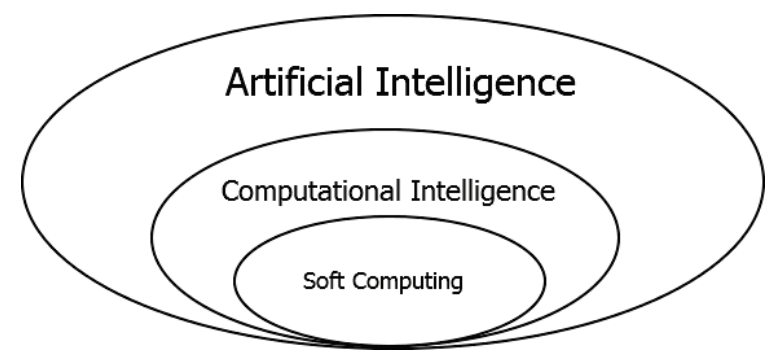

Fig. 1. Computational Intelligence in the context of soft computing and artificial intelligence. Source: Authors' own elaboration.

CI defined as a part of computer and engineering sciences devoted to solution of non-algoritmizable problems with a set of nature-inspired computational methodologies and approaches. CI community works on problems related to perception and control. It is an umbrella for three core components (fuzzy logic, artificial neural networks, and metaheuristics) [10] [16].

The basis of operation of the fuzzy logic emerges from fuzzy sets defined by Zadeh in 1965 [17]. It is defined as a fuzzy set $\boldsymbol{A}$ in a universe of discourse $\boldsymbol{U}$ that is characterized by a membership function $\mu_{(x)}$ where each domain element is assigned a degree of membership to the set in the range $[0,1]$ and is represented in the form $\mu_{(x)}: \mathbf{U} \rightarrow[0,1]$. In this way the same element can belong to several groups simultaneously only with a different degree of membership. Each fuzzy set is associated with a linguistic term that represents, for example: Low, Medium, High. See Figure 2: 


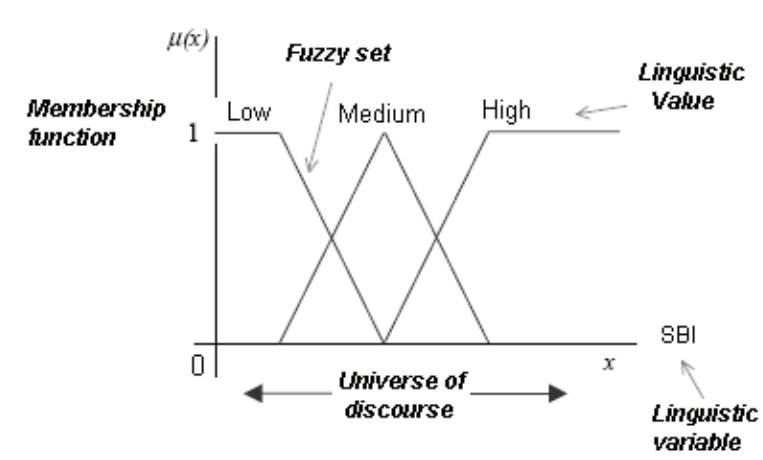

Fig. 2. Representation of terms relating to fuzzy sets.

Source: Authors' own elaboration.

Artificial neural networks (ANN) is commonly used for learning and generalization of knowledge and patterns. The ANN modeling includes the definition of three basic parameters: (1) neuron model, (2) the topology of the network and (3) the learning algorithm [18]. The Multilayer Perceptron, are most commonly used in solving complex classification problems. Figure 3 shows a view of the architecture of this type of system.

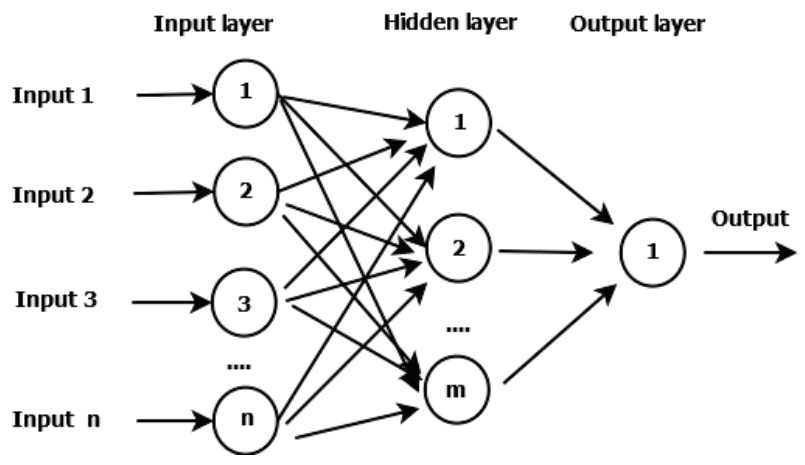

Fig. 3. Architecture of a Multilayer Perceptron.

Source: Authors' own elaboration.

Metaheuristics include methods to solve search and optimization problems inspired by the biological principles of selection, the collective intelligence of natural systems or natural genetic, such as evolutionary algorithms (EA), among others [16]. In EA, each individual is represented as a binary string or vector. Populations of competing individuals, evolve over many generations according to a fitness function. A new generation is produced by selecting the best individuals and pairing between them to produce a new set of offspring. After many generations, the descendants contain all the most promising features of a possible solution to the search problem [19]. Genetic algorithms are inserted within this context [20]. Its variants [21], have been quickly exploited as means to solving dissimilar real-world applications problems [22] [23].

In computational intelligence (like in soft computing), an individual component may be used independently. Components can also act synergistically, not competitively, to enhance an application domain of the other by integrating their individual advantages (e.g., the uncertainty handling capability of fuzzy sets, learning capability of artificial neural networks, and robust searching and optimization characteristics of metaheuristic computing). For the past 25 years in a fast way, there have been created numerous types of this kind of hybrid systems such as neuro-fuzzy [24] [25], neuro-genetic [26], genetic-fuzzy [27] [28] [29] and neuro-fuzzy-genetic [30].

The primary objective of these CI techniques is to provide more flexible information-processing systems (by using of fuzzy rules) that can exploit a tolerance for imprecision, uncertainty, approximate reasoning, learning, and partial truth, in order to achieve tractability, robustness, low solution cost, and a closer intimacy to human decision-making.

\subsection{Computational Intelligence and IT tools}

As in the case of project management, in the last decades have been developed a large number of useful tools for the application of computational intelligence techniques. The work published by Alcalá and Alonso [31], reveals a thorough study of open source-based fuzzy systems software developed as an aid to decisionmaking. In this study, a taxonomy based on two levels is presented. At the first level, three groups of jobs or solutions are met: (1) FSS general-purpose (2) specific applications and (3) fuzzy languages. Finally, on the second level, four types of contributions are grouped: (1) based on code, (2) based on libraries, (3) based on Toolbox and (4) Suite.

The application of computational intelligence techniques has had a decisive impact in other fields such as data mining [32]. Figure 4 shows the four most-used data mining tools worldwide according to a survey by KDnuggets in 2015 to 2759 voters ${ }^{\mathrm{b}}$. The survey found that only $27 \%$ of users use proprietary tools, while $73 \%$ use open source-based tools. R [33] as open sourcebased software tool is positioned in the first place followed by RapidMiner [34], which is recently considered as a commercial tool.

\footnotetext{
${ }^{\mathrm{b}}$ KDnuggets.com: [http://www.kdnuggets.com/polls/2015/analyticsdata-mining-data-science-software-used.html]
} 


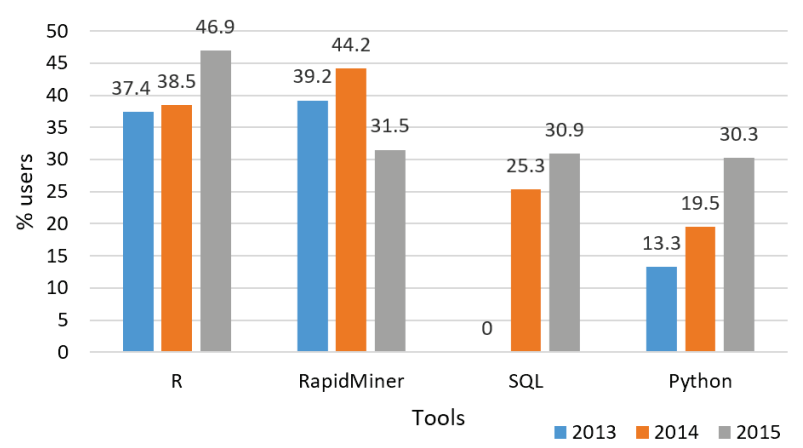

Fig. 4. Most-used data mining tools worldwide. Source: Kdnuggets ${ }^{\mathrm{b}}$.

RapidMiner is a very flexible tool that allows designing a data mining process in a visual and intuitive way, with the ease of extension from operators some of whom implemented computational intelligence techniques. It also allows ad-hoc connectivity with dissimilar types of database managers. Other tools like Weka [35], KNIME [36], KEEL [37] and MATLAB [38] also have the same facilities, through the incorporation of plugins and toolboxes respectively. However, the ad-hoc connectivity to data sources can cause inefficiencies in the information system to use, since they take into account variables such as connectivity, network speed, service availability and dependent modular architecture.

Moreover, $\mathrm{R}$ is a programming language for statistical and graphical analysis based on open source software with a high capacity for direct integration at database level through procedural languages such as PL-R [39]. R and PL-R have installation facilities both in proprietary and open source environments. $\mathrm{R}$ has a large community of developers, researchers, and users who contribute to its development by upgrading R-Cran Packages ${ }^{\mathrm{c}}$. R-Cran is a repository for storing packages or libraries with implemented algorithms ready for their application in different domains of generic or specific information. Many of these packages implement computational intelligence techniques. Among these packages, we must cite FRBS [40], SETS [41] and SAFD [42]. Finally, $\mathrm{R}$ has integrated development environments available such as R-Studio, which facilitates the incorporation of R-CRAN packages for deployment and testing in pilot projects.

Despite the existence of the discussed tools, it is not reported in specialized literature yet, the development of solutions that allow an easy integration at database level between these CI tools and IT tools to support project management. Closing this gap would provide to IT project management tools, robust mechanisms to help

${ }^{\mathrm{c}}$ R-Cran Packages: [http://cran.r-project.org/web/packages/] treat imprecision, vagueness or uncertainty in the information and improve their adaptability to handle changing management styles caused by the continuous improvement in organizations during control of their projects.

\section{Project Control and CI}

In recent years, some studies have suggested theoretical solutions based on CI, useful for project control. Specific applications of fuzzy logic, artificial neural networks, metaheuristic computing and its hybridizations in project control are relatively few compared to other application areas. Intending to provide an overview and a brief comparative analysis of the status of publications in the field of project control and CI, we use the online bibliography database ISI Web of Knowledge ${ }^{\mathrm{d}}$. As search criteria, the following queries were introduced into the link "Advanced Search":

1. Topic: Publications related to fuzzy logic and project control.

Query: TS=(fuzzy*) AND ((TS="project control*" OR $\mathrm{SO}=$ "project control*" $\mathrm{OR} \quad \mathrm{CF}=$ "project control*") OR (TS="project evaluation*" OR $\mathrm{SO}=$ "project evaluation*" $\mathrm{OR} \quad \mathrm{CF}=$ "project evaluation*") OR (TS="project assessment*" OR $\mathrm{SO}=$ "project assessment*" $\mathrm{OR} \quad \mathrm{CF}=$ "project assessment*"))

2. Topic: Publications related to neurocomputing and project control.

Query: TS $=\left({ }^{*}\right.$ neural network* OR *neuro*) AND $((\mathrm{TS}=$ "project control*" OR $\mathrm{SO}=$ "project control*" OR $\mathrm{CF}=$ "project control*") $\mathrm{OR} \quad(\mathrm{TS}=$ "project evaluation*" OR $\mathrm{SO}=$ "project evaluation*" $\mathrm{OR}$ $\mathrm{CF}=$ "project evaluation*") $\mathrm{OR} \quad(\mathrm{TS}=$ "project assessment*" OR SO="project assessment*" OR $\mathrm{CF}=$ "project assessment*"))

3. Topic: Publications related to metaheuristics and project control.

Query: TS $=(*$ heuristic* OR evolutionary* OR genetic algorithm*) AND ((TS="project control*" OR $\mathrm{SO}=$ "project control*" $\mathrm{OR} \mathrm{CF}=$ "project control*") OR (TS="project evaluation*" OR $\mathrm{SO}=$ "project evaluation*" OR $\mathrm{CF}=$ "project evaluation*") $\mathrm{OR}$ (TS="project assessment*" $\mathrm{OR} \quad \mathrm{SO}=$ "project assessment*" OR CF="project assessment*"))

In the next subsection, we make a global analysis of project control and CI visibility for each topic, regarding the amount of publications per year, associated research areas, Web of Science categories,

\footnotetext{
${ }^{\mathrm{d}}$ ISI Web of Knowledge: [http://apps.webofknowledge.com/]
} 
and trending in publications amount over a period of 21 years (from 1995 to 2015).

\subsection{Visibility at the ISI Web of Knowledge}

Research about project control and computational intelligence is definitely a growing field on which scientists are bet to incorporate information processing and machine learning techniques into the problematic situation of project control.

Figures 5, 6 and 7, shows the status of publications related to applications of fuzzy logic, neuro, and metaheuristic computing in project control over the past 21 years:

Due to the nature of hybridizations among these CI techniques and multidisciplinary approach, it is important to notice that, in some cases, the same retrieved publication can belong to more than one presented topic. Also, one publication can fit in more than one research area or Web of Science categories. That is the reason why, for research areas and Web of Science categories, the addition of values in the Record Count column of the tables does not sum up the total amount of papers returned from the corresponding query.

Obtained analytics from the presented topics, shows that regarding publications amount, the most worked field has been the one related to applications of fuzzy logic (126), following by metaheuristics (30) and ending in neurocomputing, with a close gap of publications amount difference (from 28). Concerning the amount of publications per year, we can see that recent peaks occur in 2010, 2012 and 2009 respectively for each presented topic. Thus, a superficial trend about which is the latest worked CI approach to project control could be inferred, corresponding to neurocomputing. However, globally, the number of recent publications suggests again fuzzy logic as the most proposed CI technique, with 11 publications in 2015 .

In addition, notice that, regardless of which CI technique approach is used, the most benefited research areas have been those related to Engineering and Computer Sciences. Despite this apparently "benefit", the true meaning of this, is the challenge situation would be related about how to increase the application range of future research towards more practical fields on project management, like Transportation, Energy Fuels, Physics, Telecommunications, Business Economics, etc.

In the matter of Web of Science categories, the main developed concepts in all of these publications logically fall within Computer Science and Artificial Intelligence. Besides, it is worthy to note that in developed works, there is involved a significant evidence pointing out to practical application areas like Civil, Industrial and Electronics Engineering. But again, the necessity of incorporating more related categories to given proposals is a call to be "a must" for future prospects in the debated field.

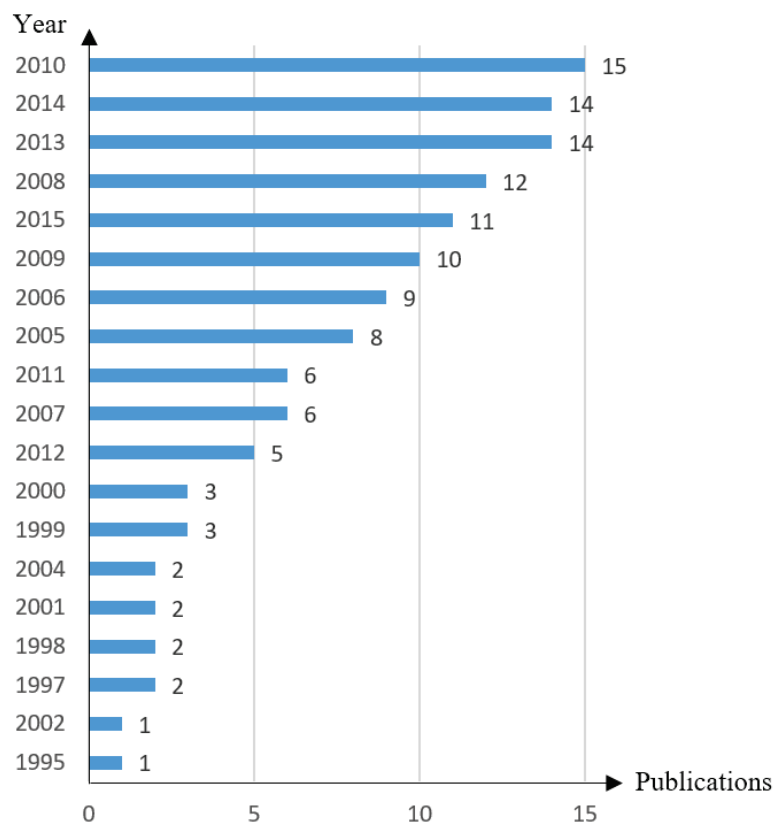

(a) Publications per year.

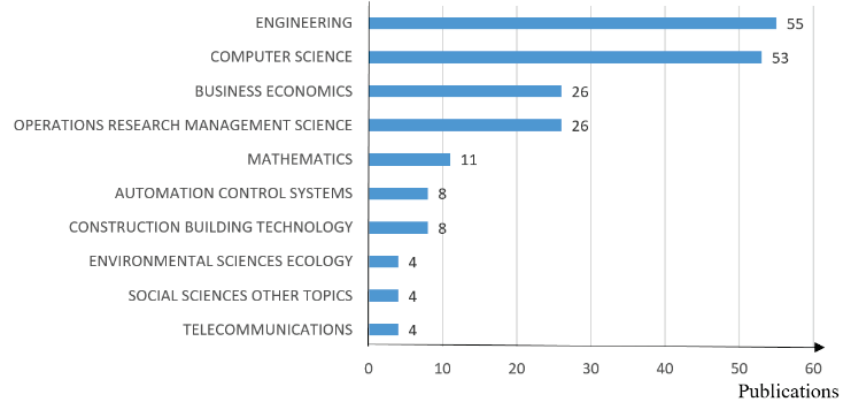

(b) Research Areas (top-10).

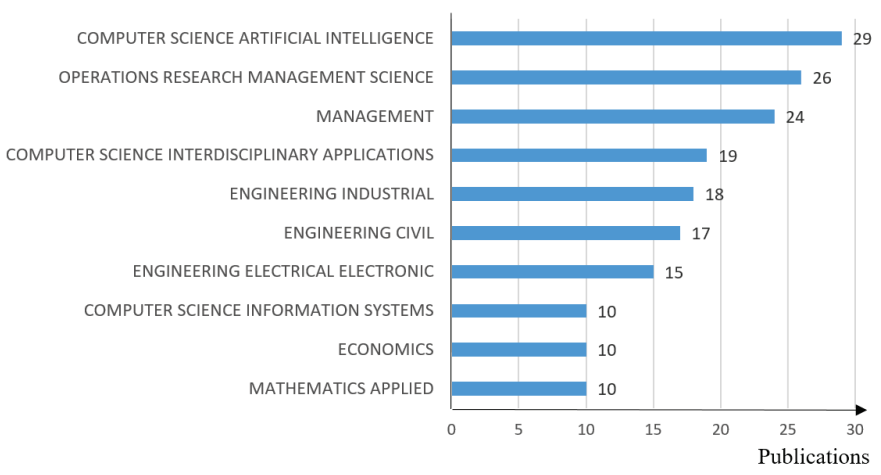

(c) Web of Science Categories (top-10)

Fig. 5. Query (1). Topic: publications related to fuzzy logic and project control during the last 21 years. Data (October 17, 2016) retrieved from the ISI Web of Knowledge. 


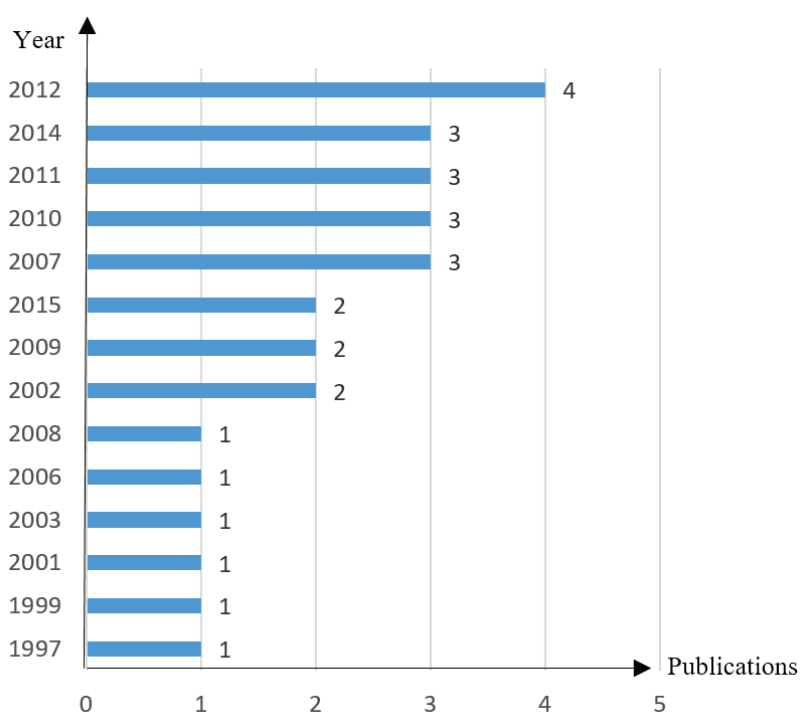

(a) Publications per year.

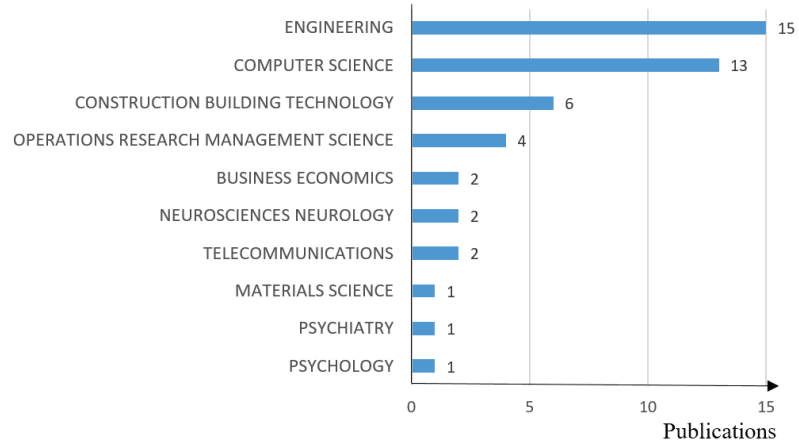

(b) Research Areas (top-10).

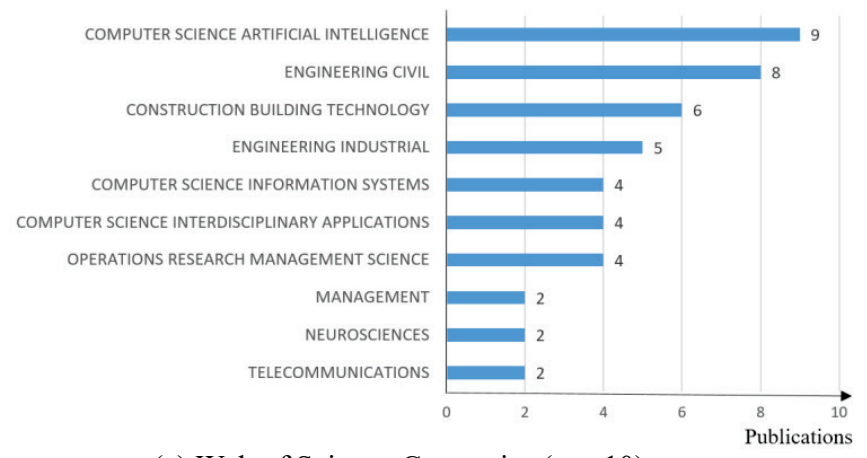

(c) Web of Science Categories (top-10).

Fig. 6. Query (2). Topic: publications related to neurocomputing and project control during the last 21 years. Data (October 17, 2016) retrieved from the ISI Web of Knowledge.

In order to analyze the interest trending from the international research community regarding project control and CI over a period of 21 years, we split the

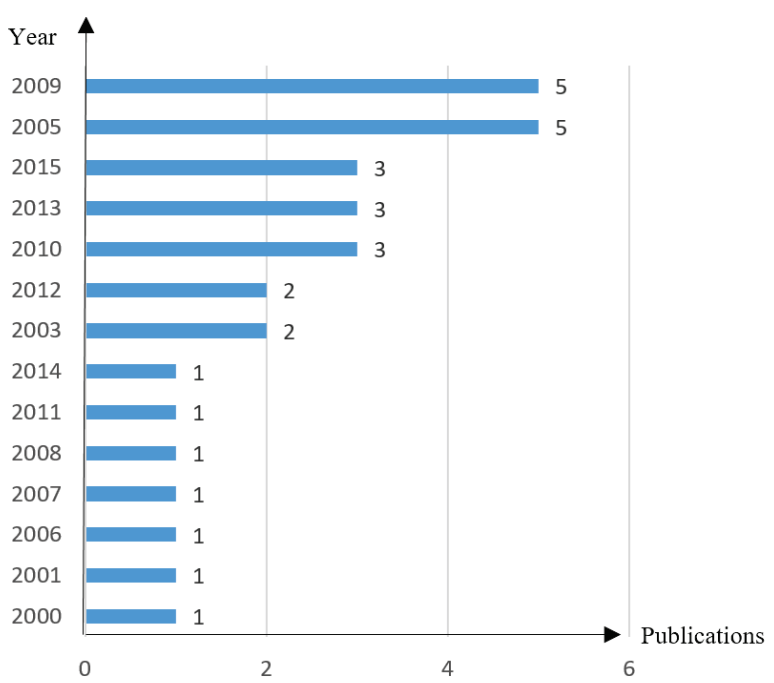

(a) Publications per year.

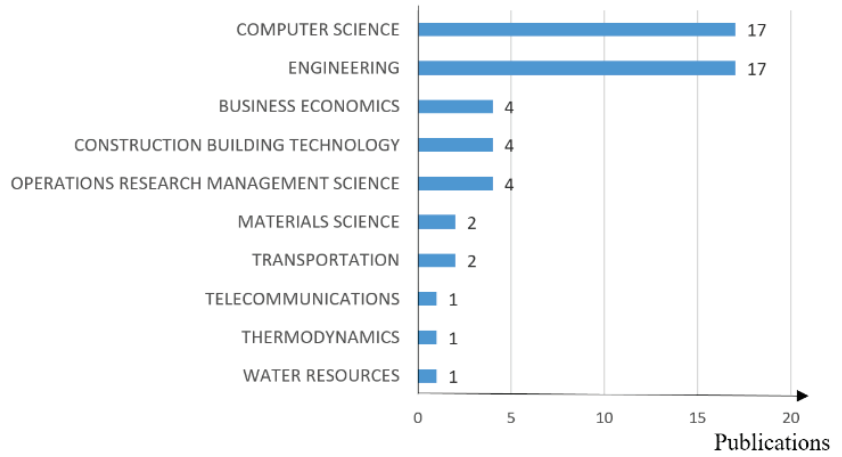

(b) Research Areas (top-10).

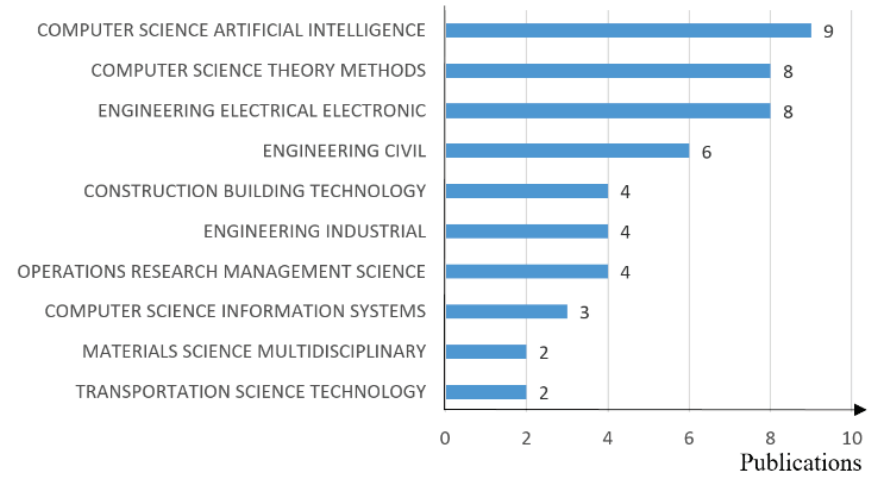

(c) Web of Science Categories (top-10).

Fig. 7. Query (3). Topic: publications related to metaheuristic computing and project control during the last 21 years. Data (October 17, 2016) retrieved from the ISI Web of Knowledge.

number of existing publications chronologically in three approximately equal periods. For data processing, the Scimat [43] software tool was used. Figures 8, 9 and 10 shows the corresponding information by topic. 


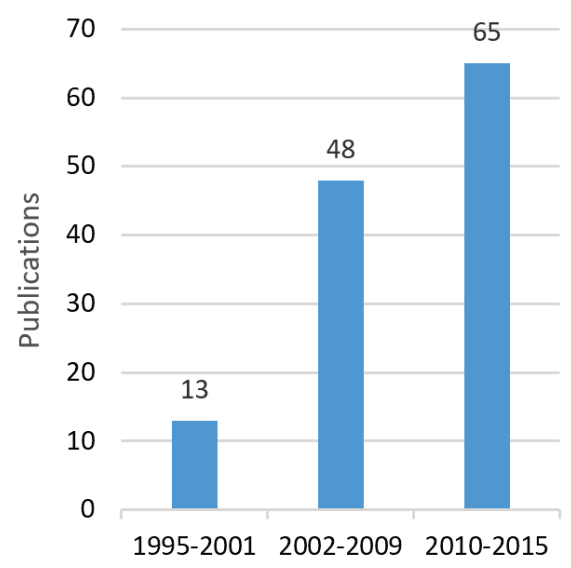

Fig. 8. Interest trending related to fuzzy logic and project control over the last 21 years. Data (October 17, 2016) produced by Scimat [43].

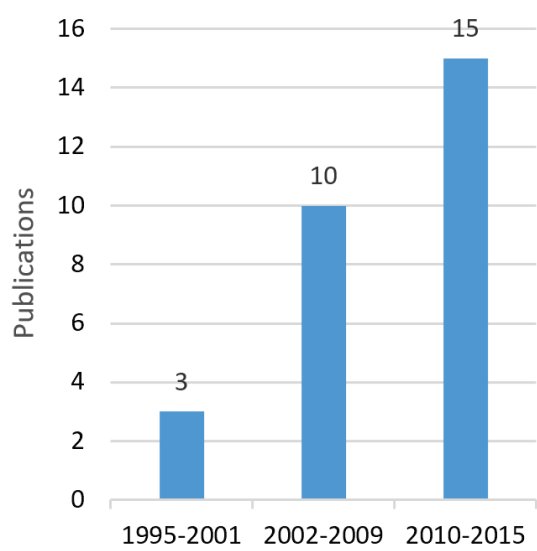

Fig. 9. Interest trending related to neurocomputing and project control over the last 21 years. Data (October 17, 2016) produced by Scimat [43].

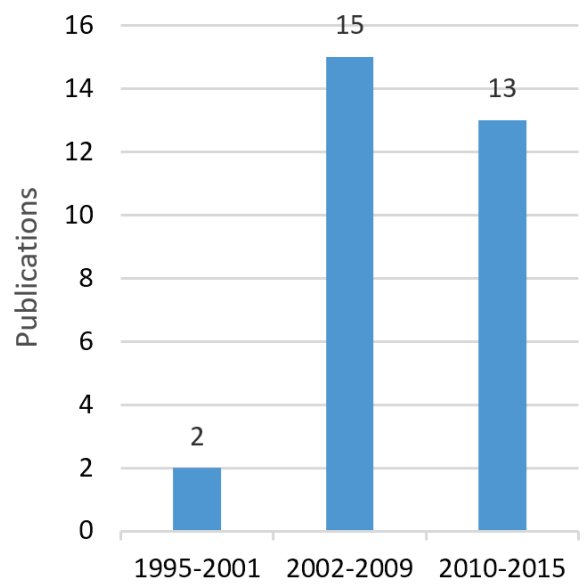

Fig. 10. Interest trending related to metaheuristic computing and project control over the last 21 years. Data (October 17, 2016) produced by Scimat [43].
Although metaheuristic computing proposals have experimented a slight decrease of published papers in the last 6 years, we can see the increasingly raise up of the use of fuzzy logic and neurocomputing techniques within project control problems over the last two decades. This periodical growth confirms the proven effectiveness of fuzzy logic, neurocomputing and its hybridizations into the field of control application problems. Thus, it settles the rising fact that there is an associated valuable niche market and prospective benefit around the application of these specific approaches within the context of project control applications. Nevertheless, this does not mean metaheuristic computing must be disregarded, because depending on nature of raw data and No Free Lunch Theorem [44], could be still a valuable approach for some kind of problems.

\subsection{Some of the most representative works in the field of project control and $C I$}

With the aim of finding out the content and gaps of current research trends, this subsection examines works published in the last ten years (from 2006 to 2015). Also, although project control is a very broad term and there exists many kind of projects $(\mathrm{R} \& \mathrm{D}$, investment, financial, construction, etc.), for the sake of clarity in this investigation, we only intent to analyze those papers which helps to provide some kind of generic solutions to all kind of projects. The presented papers were selected according to their contribution degree to the resolution of the deficiencies identified in the problematic situation of this investigation. Therefore, the focus on the analysis falls on four aspects: (1) proposed indicators and coverage of knowledge areas, (2) treatment of imprecision, vagueness or uncertainty, (3) optimization/adjustment of the inference system, and (4) integration with IT project management tools. Next, the selected papers are presented.

Dweiri and Kablan [45] combine cost, time and quality measures to obtain the internal efficiency of the project development. The fuzzy decision-making system proposed for evaluating the efficiency is implemented via MATLAB. This is a static model where the rules are manually introduced into the system and the fuzzy sets of input and output variables are based on the experience and knowledge of human experts. A very similar and more recent work, although using only input indicators associated with time, it is the proposed by Kumar and Krishan [46]. Both studies illustrate applications through a case of study, but lacking of optimization mechanisms for the set of rules and integration with IT project management tools in real work production environments. 
Lee et al. [47] propose an ontology-based intelligent decision support agent (OIDSA) for CMMI project monitoring and control. The OIDSA is composed of a natural language processing agent, a fuzzy inference agent, and a performance decision support agent. All the needed information of the OIDSA, including the CMMI ontology and the project personal ontology, is stored in an ontology repository. In addition, the natural language processing agent, based on the Chinese Dictionary, periodically collects the information of the project progress from project members to analyze the features of the Chinese terms for semantic concept clustering. Next, the fuzzy inference agent computes the similarity of the planned progress report and actual progress report, based on the CMMI ontology, the project personal ontology, and natural language processing results. Finally, the performance decision support agent measures the completed percentage of the progress for each project member. However, the proposal only shows experimental results. Also, lack of mechanisms of integration with IT project management tools in real work production environments. Moreover, has deficiencies in components for optimization or adjustment of the rules on the fuzzy inference agent used.

Bath [48] proposed a soft computing-based method to classify projects into three classes (simple, medium or complex). There can stand out as interesting, the proposed data pre-processing phase to remove irrelevant information, the feature extraction phases (input variables) and their relevance analysis. However, these theoretical and experimental contributions, do not provide solutions to the research problem involved with project control by the use of key performance indicators that cover main knowledge areas.

Gao [49], created an indexing system is to evaluate the performance in project management. This is treated as a problem of multi-criteria decision by combining the fuzzy theory and analytical network process (ANP) to provide a solution. They take into account the quality, cost, time and risk indices. However, they don't include other KPIs like, for example, human resources, logistics or integration. In this research techniques for learning and adjusting the fuzzy sets parameters are not included, which affects the efficacy of the system for project evaluation according to continuous improvement in management styles of the organization which employs it.

Cheng et al. [50] [51] have an evolutionary-fuzzy hybrid neural network for project cash flow control. Promote the use of a model that receives as input data indicators on cash operations. Then these data are represented by fuzzy sets, which come to an inference system rules-based. The rule generation is optimized by a hybrid based on genetic algorithms and neural network approach. Finally, the system shows the expected output. Being a model that provides particular solution for project cash flow control problems, does not handle as inputs using other key performance indicators on fundamental knowledge areas for project control. It also lacks mechanisms of integration with IT project management tools in real work production environments.

The work of Li and Guo [52], proposes a hybrid approach based on the use of genetic algorithms and neural networks to evaluate the maturity of project management in construction companies. It suggests using a functional platform-RNA based on a genetic model, and a set of indicators for the assessment of this maturity. Although not explicitly defined, the proposed indicators associates with fundamental knowledge areas of project management. However, the proposed approach does not make the treatment of imprecision contained in the information systems. Nor it promotes the generation of fuzzy rules for the purpose of evaluating the execution of the project, which affects aid effectiveness for decision-making.

Certa [53] proposed a synthetic measure for the assessment of the project performance, combining two inputs obtained by the earned value analysis (cost performance and performance planning). It takes into account eight fuzzy sets for the input linguistic variables and seven for the output obtaining 64 fuzzy rules covering all combinations of input variables. The fuzzy sets are represented with triangular and trapezoidal functions, taking positive and negative values; the parameters of the functions are defined by experts. Three very similar research that applied the earnedvalue-fuzzy approach are also presented by Moslemi [54] [55] and Salari [56]. On these papers, a linguistic representation for the indicators used are shown. While these investigations constitute a concrete theoretical contribution, lack of mechanisms for creation/generation of the initial set of fuzzy rules. Also, does not analyze other KPIs related with fundamental areas of project management, such as human resources or logistics, equally significant when controlling the project.

In the Ph.D. dissertation of McQuighan [57], a computational intelligence approach for project scope control is presented. They propose the use of indicators related with time and cost in order to measure de project scope constraints. This research looks at the fuzzy nature of the inputs to status reports for the scope constraint to answer two questions: (1) Can fuzzy systems offer a tool that can capture the status of the scope of an individual activity in an IT project? and (2) Can the scope status for activities be aggregated into a 
meaningful project scope status? The Z-mouse tool proposed by Zadeh, which is an extension of Zadeh's work on fuzzy set theory [58], is a leading-edge data collection mechanism that is being used as the data collection tool in this study. In this research, techniques for learning and adjusting the fuzzy sets parameters are not included. Also, because it is directed to the control of project scope, leaves out other important knowledge areas for project control.

In the work of Lee et al. [59], a reasoning process is presented as support for integrated project control. It proposes as entries, the use of well-known Cost Performance Index (CPI), Schedule Performance Index (SPI) and Earned Value indicators. The reasoning process is achieved in a prototype web application system, improving efficiency in assisting decision making through integrated project control. However, the proposal lacks the inclusion of other key indicators for project control, such as human resources or logistics. Nor does it suggest the use of mechanisms for generating rules that allow adjustment of project evaluation system according to the continuous improvement in management styles of the environment where it is used.

In the work of Hammel et al. [60] it is proposing a study related to computational intelligence for information technology project management. The research is intended to project managers who seek more robustness tools to deal in problem domains in which information, data and perhaps even the problem itself are vague, approximate, and uncertain. Recognizes the increasing complexity of today's IT projects, the need for intelligent tools, processes, methods, and systems to assist in the management of these projects is also growing. Despite this, the study is primarily theoretical and only provides knowledge about how to apply the CI core technologies but not offer practical solutions to real life or to specific scenario for project control applications.

Mewada et al. [61] presents an evaluation model of software effort using an Adaptive Neuro-Fuzzy Inference System (ANFIS) on uncertain data. With this technique, the results in predictions turn out to be very good and even better than those obtained from applying other techniques like neural networks. Provides a brief overview of the application of traditional techniques and soft computing in evaluating software effort. For implementing the experiments, it is used MATLAB over a total of 18 NASA projects as dataset. Although this work provides some generic solution principles which could be valuable for project control problems, the model is not applied for this purpose.

In the paper of Liu et al. [62], a research on Evaluation of Project Management Maturity Model
Based on BP Neural Network is proposed. The system is trained and validated with MATLAB. Results shows that it is possible to obtain an accurate and objective assessment; however, these results are not integrated with IT project management tools.

On the research of Govindarajan [63] a fuzzy inference system for evaluating the efficiency in the management of software projects is proposed. The Fuzzy Logic toolbox of MATLAB and Simulink are used for the implementation. They use four triangular functions for each fuzzy set in the two input variables ("project delay" and "Change in project delay") and the output variable ("Software project management efficiency"). As a future work, they suggest the use of ANFIS to improve the fuzzy inference system created, which is static.

Early studies about using neuro-fuzzy for project control, are the proposed by Vasappanavara et al. [64] and $\mathrm{Yu}$ at al. [65]. The authors establish a research around information systems in the context of project performance evaluation based on fuzzy neural network approach. More recently, in the work of Hossein [66], a new simulation approach it is proposed to develop project progress time-series data, based on the complexity and specifications of the project as well as on the environment in which the project is executed. This study provides researchers with an opportunity to generate general and customized methods such as project completion time estimation. Thus, it is used ANN, ANFIS, Emotional Learning based Fuzzy Interface System (ELFIS) and Conventional Regression to forecast the completion time of project. Although all of these works used KPIs related to time and cost management as input for the project control evaluation system such as SPI and CPI for the EV calculation, does not include other key performance indicators, such as human resources or logistics. Also, lacks of integration mechanisms with IT project management tools in real time work production environments.

\section{Critical Remarks and Further Challenges}

In recent years, an increasing corpus of research has been developed in the area of project control and CI. The previous subsection just presented what is already done and the apriori necessities. But, what should be done in the short and mid-term? how to address still open problems and challenges? The analyzed research and project management schools demonstrate the necessity of constructing new models and tools for project control by using CI. But this issue must be engaged in a way which integrates all the following directions: 
- Automated calculation of indicators based on evidence (measures) taken as a result of the production development process, covering the key knowledge areas of project management (time, cost, quality, logistics and human resources performance).

Team members should not type measures excessively into the PMIS. In order to not interfering with production environment and in cases on which can be applied, some measures can be gathered using sensors with CI programming interfaces. These measures could be (but not limited to) those related to time scheduling (percentage of execution completion), human resource assessment (task evaluation) or logistic performance (assets valuation).

- Application of machine learning-based approaches with the treatment of imprecision, vagueness or uncertainty in the information.

The amount of CI-based machine learning algorithms out there yields to another challenging question: How do we know which is better and when to use it? Knowledge bases for PMIS under operation should be deployed first. Preceded by a proper sampling technique to select finished projects, calculated performance indicators associated with key knowledge areas and expert's evaluation for each finished project must be present. Then, we need to select a set of prospective algorithms to be tested over the knowledge base. The selection could be performed according to some specific interest or taking a random sample of algorithms among each CI approach (fuzzy, neuro, metaheuristics or hybrid). With the aim of finding out which algorithm performs best, proper validation steps need to be done. As a starting point, the research of Demšar [67] and Derrac e et al. [68] [69] provide meaningful insights regarding this topic. The automation and development of integrated validation mechanisms for CI application into PMIS, bring new opportunities in the future.

- Adjustment of the evaluation system according to the continuous improvement in the organization management styles.

Organizations are continuously changing. Adaptive-project-control systems will be needed. New KPIs to be use or deleted, changing of expert's evaluation rules, are commonly known scenarios among project managers. It is essential for PMIS handle that kind of situations. Establish well-defined

\footnotetext{
${ }^{\mathrm{e}}$ More info at Soft Computing and Intelligence Information Systems, University of Granada research group: [http://sci2s.ugr.es/sicidm]
}

automated processes between high management levels of the organization and the PMIS internal settings, promise new relationship features in the way on which these two entities communicate between them.

- Model integration with IT project management tools based on open source software.

Despite the large number of IT project management tools, open source software for project control is still few compared to its proprietary counterpart. The integration of CI techniques at core and database level would provide an immediate solid competition on the field of PMIS.

These critical remarks emphasize the fact that project control and CI is today a growing field with vast opportunities towards new research and development areas. Emergent ISI Web of Science categories remarks the necessity of keep going further, focusing on more practical solutions within the project management discipline. Finally, in order to meet future requirements, project management schools need to be continuously reviewed at the time when new $\mathrm{CI}$ methods will be incorporated.

\section{Conclusions}

This paper investigates the trends and challenges in project control and computational intelligence. The study makes a contribution towards identifying niche markets with high applicability around the thematic goal. Also, it was possible to reach the following conclusions:

- Project control is a complex task that involves vagueness in concepts and uncertainty in information, a situation where the use of $\mathrm{CI}$ techniques yields good results.

- The use of machine learning methods into CI techniques for the evaluation of projects increases the adaptability of organizations in front of the changing management styles caused by the maturity reached during its continuous improvement.

- The implementation of new libraries and associated methodologies for learning evaluation in project control with open source software tools and programming languages such as PL-R, opens a field of research related to increase technological integration with IT project management tools.

- The content under discussion provides support to improve decision-making in project-oriented organizations. 


\section{References}

1 PMI. Project Management Body of Knowledge (PMBOK), 5th edn. (PMI Publications, Pennsylvania, 2013).

2 ISO. ISO 21500:2012 Guidance on Project Management, (International Organization for Standardization, 2012).

3 The Standish Group. The CHAOS Manifesto. Think Big, Act Small, (The Standish Group, 2013).

4 L. Pacelli. The Project Management: 18 Major Project Screw-Ups, and How to Cut Them Off at the Pass, (Prentice Hall, New Jersey, 2004), pp. 23-29.

5 K. Jugdev, D. Perkins, J. Fortune, D. White and D. Walker. An exploratory study of project sucess with tools, software and methods. International Journal of Managing Projects in Business, 6(3):534-551, 2012.

6 M. Caniëls and R. Bakens. The effects of Project Management Information Systems on decision making in a multi project environment. International Journal of Project Management, 30(2):162-175, 2012.

7 A. Botchkarev and P. Finnigan. Complexity in the context of information systems project management. Organisational Project Management, 2(1):15-34, 2015.

8 H. Emdon. How does Open Source Software contribute to socio-economic development?. Master thesis, University of the Witwatersrand, Johannesburg, 2010.

9 R. Bello and J. L. Verdegay. Rough sets in the Soft Computing environment. Information Sciences, 212:1-14, 2012.

10 W. Duch. What is Computational Intelligence and Where Is It Going? in W. Duch, J. Mańdziuk (Eds.), Challenges for Computational Intelligence, volume 63 of Studies in Computational Intelligence (Springer-Verlag, Berlin, 2007), pp. 1-13.

11 L. Magdalena. What is Soft Computing? Revisiting Possible Answers. International Journal of Computational Intelligence Systems, 3(2):148-159, 2010.

12 PRINCE2. Managing Successful Projects with PRINCE2, (Renouf Pub Co Ltd, London, 2009), pp. 99-109.

13 SEI. CMMI for Dev v1.3., (Carnegie Mellon University, Pittsburgh, 2010), pp. 127-363.

14 D. Stang, R. Handler and T. Jones. Magic Quadrant for Cloud-Based IT Project and Portfolio Management Services, Worldwide. (Gartner Inc., Stamford, 2015).

15 D. Stang, R. Handler and T. Jones. Magic Quadrant for IT Project and Portfolio Management Software Applications, Worldwide. (Gartner Inc., Stamford, 2015).

16 J. L. Verdegay, R. R. Yager and P. P. Bonissone. On heuristics as a fundamental constituent of soft computing. Fuzzy Sets and Systems, 159:846-855, 2008.

17 L. A. Zadeh. Fuzzy Sets. Information and Control, 8(3):338-353, 1965.

18 B. Yegnanarayana, Artificial Neural Networks, $3^{\text {rd }}$ edn. (PHI Learning Pvt. Ltd., New York, 2009).

19 D. Simon. Evolutionary optimization algorithms. Biolocally Inspired and Population-Based Approaches to
Computer Intelligence, (John Wiley \& Sons, Inc., New Jersey, 2013).

20 M. Mitchell, An Introduction to Genetic Algorithms, (MIT Press, Cambridge, MA, 1996).

21 S. M. Elsayed, R. A. Sarker and D. L. Essam. A comparative study of different variants of genetic algorithms for constrained optimization, in Simulated Evolution and Learning, (Springer-Verlag, Berlin, 2010), pp. 177-186.

22 R. Chiong, T. Weise and Z. Michalewicz. Variants of Evolutionary Algorithms for Real-World Applications, $1^{\text {st }}$ edn. (Springer-Verlag, Berlin, 2012).

23 A. Fernández, V. López, M. J. del Jesus and F. Herrera. Revisiting Evolutionary Fuzzy Systems: Taxonomy, applications, new trends and challenges. KnowledgeBased Systems, 80:109-121, 2015.

24 J. R. Jang. ANFIS: Adaptive-Network-Based Fuzzy Inference System. IEEE Transaction Systems Man \& Cybernetics, 23(3):665-685, 1993.

25 D. Nauck, F. Klawonn and R. Kruse. Foundations of Neuro-Fuzzy Systems (John Wiley \& Sons, Inc., New Yor, 1997).

26 P. Koehn. Combining Genetic Algorithms and Neural Networks: The Encoding Problem. Master thesis, The University of Tennessee, Knoxville, 1994.

27 F. Herrera, M. Lozano and J. L. Verdegay. A learning process for fuzzy control rules using genetic algorithms. Fuzzy Sets and Systems, 100:143-158, 1998.

28 O. Cordon, M. J. del Jesus, F. Herrera and M. Lozano. MOGUL: A methodology to obtain genetic fuzzy rulebased systems under the iterative rule learning approach. International Journal of Intelligent Systems, 14(11):11231153, 1999.

29 R. Alcalá, J. Alcalá and F. Herrera. A proposal for the genetic lateral tuning of linguistic fuzzy systems and its interaction with rule selection. IEEE Transactions on Fuzzy Systems, 15(4):616-635, 2007.

30 I. Djelloul. Neuro-Fuzzy Genetic Algorithms for monitoring in a production system, in 3rd International Conference on Systems and Control (ICSC), eds. IEEE (Algiers, 2013), pp. 12-17.

31 J. Alcalá-Fdez and J. M. Alonso. A survey of fuzzy systems software: taxonomy, current research trends and prospects. IEEE Transactions on Fuzzy Systems, 24(1):40-56, 2015.

32 F. Neukart, C. Grigorescu and S.-A. Moraru. High Order Computational Intelligence in Data Mining: A generic approach to systemic intelligent Data Mining, in 6th Conference on Speech Technology and Human-Computer Dialogue, (Brasov, 2011), pp. 1-9.

$33 \mathrm{R}$ Core Team. R: A Language and Environment for Statistical Computing, (R Foundation for Statistical Computing, Vienna, Austria, 2015).

34 M. Hofmann and R. Klinkenberg. Rapidminer: Data Mining Use Cases and Business Analytics Applications, (Chapman \& Hall/CRC, Boca Ratón, FL:, 2013). 
35 I. H. Witten, E. Frank and M. A. Hall. Data mining: Practical machine learning tools and techniques, $3^{\text {rd }}$ edn. (Morgan Kaufmann, San Francisco, 2011).

36 M. R. Berthold, B. Wiswedel and T. R. Gabriel. Fuzzy logic in KNIME, modules for approximate reasoning. International Journal of Computational Intelligence Systems, 6(Suppl.):34-45, 2013.

37 J. Alcalá-Fdez, A. Fernández, J. Luengo, J. Derrac, S. García, L. Sánchez and F. Herrera. KEEL Data-mining software tool: data set repository, integration of algorithms and experimental analysis framework. Journal of Multiple-Valued Logic and Soft Computing, 17(23):255-287, 2011.

38 Mathworks. MATLAB, Fuzzy Logic Toolbox, User's Guide, (The Mathworks Inc., Massachusetts, 2014).

39 M. Basille, F. Urbano and J. Conway. A step further in the integration of data management and analysis: $\mathrm{Pl} / \mathrm{R}$, in Spatial Database for GPS Wildlife Tracking Data, eds. F. Urbano and F. Cagnacci (Springer International Publishing, Berlín, 2014), pp. 213-229.

40 L. S. Riza, C. Bergmeir, F. Herrera and J. M. Benitez. frbs: Fuzzy Rule-Based Systems for Classification and Regression in R. Journal of Statistical Software, 65(6):130, 2015 .

41 D. Meyer and K. Hornik. Generalized and customizable sets in R. Journal of Statistical Software, 31(2):1-27, 2009.

42 W. Trutschnig, M. A. Lubiano and J. Lastra. SAFD - an $\mathrm{R}$ package for statistical analysis of fuzzy data, in Towards Advanced Data Analysis by Combining Soft Computing and Statistics, eds. C. Borgelt, M. Gil, J. Sousa and M. Verleysen (Springer, Berlín, 2013), pp. 107-118.

43 M. J. Cobo, A. G. López-Herrera, E. Herrera-Viedma and F. Herrera. SciMAT: A new science mapping analysis software tool. Journal of the American Society for Information Science and Technology, 63(8):1609-1630, 2012.

44 W. A. Dembski. No Free Lunch. Why Specified Complexity Cannot be Purchased Without Intelligence, $1^{\text {st }}$ edn. (Rowman and Littlefield Publishers, Maryland, 2002).

45 F. T. Dweiri and M. M. Kablan. Using fuzzy decision making for the evaluation of the project management internal efficiency. Decision Support Systems, 42:712726, 2006.

46 V. Kumar and S. Krishan. Project management efficiency - A fuzzy logic approach. International Journal of Engineering and Advanced Technology, 1(3):34-38, 2012.

47 C.-S. Lee, M.-H. Wang and J.-J. Chen. Ontology-based intelligent decision support agent for CMMI project monitoring and control. International Journal of Approximate Reasoning, 48(1):62-76, 2008.

48 M. Bhatt. Project Classification Using Soft Computing, in International Conference on Advances in Computing,
Control, \& Telecommunication Technologies, ACT '09. (Trivandrum, Kerala, 2009).

49 H. Gao. A fuzzy-ANP approach to project management performance evaluation indices system, in 2010 International Conference on Logistics Systems and Intelligent Management, (Harbin, 2010), pp. 273-277.

50 M.-Y. Cheng, H.-C. Tsai and E. Sudjono. Evolutionary fuzzy hybrid neural network for project cash flow control Engineering Applications of Artificial Intelligence, 23(4):604-613, 2010.

51 M.-Y. Cheng and A. Roy. Evolutionary fuzzy decision model for cash flow prediction using time-dependent support vector machines. International Journal of Project Management, 29(1):56-65, 2011.

52 J. Li and L. Guo. A Research on Evaluating Project Management Maturity Based on Genetic BP Neural Network Data Mining Method, in International Conference on Artificial Intelligence and Computational Intelligence, (Sanya, 2010), pp. 477-480.

53 A. Certa, M. Enea and A. Giallanza. A Synthetic Measure for the Assessment of the Project Performance, in Business Performance Measurement and Management, ed. P. Taticchi, (Springer-Verlag, Berlin, 2010), pp. 167-180.

54 L. Moslemi and A. Salehipour. Evaluating fuzzy earned value indices and estimates by applying alpha cuts. Expert Systems with Applications, 38(7):8193-8198, 2011.

55 L. Moslemi, S. Shadrokh and A. Salehipour. A fuzzy approach for the earned value management. International Journal of Project Management, 29(6):764-772, 2011.

56 M. Salari, M. Bagherpour and M. H. Reihani. A time-cost trade-off model by incorporating fuzzy earned value management: A statistical based approach. Journal of Intelligent \& Fuzzy Systems, 28(4):1909-1919, 2015.

57 J. M. McQuighan. A fuzzy computational intelligence approach to monitoring information technology project scope. PhD. thesis, Towson University, Baltimore, 2013.

58 Zadeh, L.A. Outline of A New Approach to the Analysis of Complex Systems and Decision. IEEE Transactions on Systems, Man, and Cybernetics, 3(1):28-44, 1973.

$59 \mathrm{~J}$. Li, O. Moselhi and S. Alkass. A reasoning process in support of integrated project control. Canadian Journal of Civil Engineering, 32(3):500-516, 2011.

60 R. J. Hammell, J. Hoksbergen, J. Wood and M. Christensen. Computational Intelligence for Information Technology Project Management, in Machine Learning: Concepts, Methodologies, Tools and Applications, (IGI Global, Hershey, PA, 2012), pp. 1601-1624.

61 K. M. Mewada, A. Sinhal and B. Verma. Adaptive Neuro-Fuzzy Inference System (ANFIS) Based Software Evaluation. International Journal of Computer Science, 10(1):244-250, 2013.

62 Y. Liu, D. Zheng and E. Wang. Research on Evaluation of Project Management Maturity Model Based on BP Neural Network. Advances in information Sciences and Service Sciences, 5(2):693-701, 2013. 
63 A. Govindarajan. A Novel Framework for Evaluating the Software Project Management Efficiency - An Artificial Intelligence Approach. TELKOMNIKA Indonesian Journal of Electrical Engineering, 12(9):7054-7058, 2014.

64 G. Vasappanavara, A. Vasappanavara, D. Ravi and R. Vasappanavara. Special tutorial - Project management: Issues in computer based monitoring and control with soft computing approaches, in Applications of Soft Computing (Berlin, Springer-Verlag, 2006), pp. 415-420.

65 B. Yu, J. Zhang and S. Wei. The Research on Information System Project Performance Evaluation Based on Fuzzy Neural Network, in 3rd International Conference on Wireless Communications, Networking and Mobile Computing (Shanghai, 2007), pp. 6170-6174.

66 S. Hossein and Z. Tavassoli. Intelligent Systems in Project Performance Measurement and Evaluation, in Intelligent Techniques in Engineering Management, (Springer International Publishing, Switzerland, 2015), pp. 581-619.
67 J. Demšar. Statistical comparisons of classifiers over multiple data sets. Journal of Machine Learning Research, 7:1-30, 2006.

68 J. Derrac, S. García, D. Molina and F. Herrera. A practical tutorial on the use of nonparametric statistical tests as a methodology for comparing evolutionary and swarm intelligence algorithms. Swarm and Evolutionary Computation, 1(1):3-18, 2011.

69 J. Derrac, S. García, S. Hui, P. Nagaratnam-Suganthan and F. Herrera. Analyzing convergence performance of evolutionary algorithms: A statistical approach. Information Sciences, 289:41-58, 2014. 
Appendix A

\begin{tabular}{|c|c|c|c|c|c|}
\hline Proj & ect Management Information System & 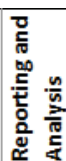 & 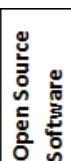 & 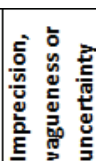 & 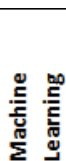 \\
\hline 1 & Project.NET & Yes & Yes & No & No \\
\hline 2 & Project-Open & Yes & Yes & No & No \\
\hline 3 & OpenERP & Yes & Yes & No & No \\
\hline 4 & AtTask & Yes & No & No & No \\
\hline 5 & Clarizen & Yes & No & No & No \\
\hline 6 & Genius Inside & Yes & No & No & No \\
\hline 7 & Microsoft Office Project Server & Yes & No & No & No \\
\hline 8 & Planisware 5 & Yes & No & No & No \\
\hline 9 & Primavera Project Planner & Yes & No & No & No \\
\hline 10 & Projektron BCS & Yes & No & No & No \\
\hline 11 & PSNext & Yes & No & No & No \\
\hline 12 & Severa & Yes & No & No & No \\
\hline 13 & Tenrox & Yes & No & No & No \\
\hline 14 & TrackerSuite.Net & Yes & No & No & No \\
\hline 15 & WorkLenz & Yes & No & No & No \\
\hline 16 & Assembla & Yes & No & No & No \\
\hline 17 & QuickBase & Yes & No & No & No \\
\hline 18 & 24SevenOffice & Yes & No & No & No \\
\hline 19 & EPM Live & Yes & No & No & No \\
\hline 20 & Compuware Changepoint & Yes & No & No & No \\
\hline 21 & Rally Software & Yes & No & No & No \\
\hline 22 & TeamDynamixHE & Yes & No & No & No \\
\hline 23 & in-Step & Yes & No & No & No \\
\hline 24 & $5 \mathrm{pm}$ & Yes & No & No & No \\
\hline 25 & Plandora & Yes & Yes & No & No \\
\hline 26 & OnTime & Yes & No & No & No \\
\hline 27 & enQuire & Yes & No & No & No \\
\hline 28 & Eclipse PPM software & Yes & No & No & No \\
\hline 29 & Celoxis & Yes & No & No & No \\
\hline 30 & CA Clarity PPM & Yes & No & No & No \\
\hline 31 & PLANTA Project & Yes & No & No & No \\
\hline 32 & Endeavour Software Project & No & Yes & No & No \\
\hline 33 & Merlin & Yes & No & No & No \\
\hline 34 & Oracle Project Portfolio Management & Yes & No & No & No \\
\hline 35 & workspace.com & Yes & No & No & No \\
\hline 36 & Redmine & No & Yes & No & No \\
\hline 37 & Copper Project & Yes & No & No & No \\
\hline 38 & MyWorkPLAN & Yes & No & No & No \\
\hline 39 & NetSuite & Yes & No & No & No \\
\hline 40 & Webforum Project & Yes & No & No & No \\
\hline 41 & Journyx & No & No & No & No \\
\hline 42 & SAP RPM & No & No & No & No \\
\hline 43 & Teamwork PM & No & No & No & No \\
\hline 44 & eGroupWare & No & Yes & No & No \\
\hline 45 & FogBugz & Yes & No & No & No \\
\hline 46 & WorkPLAN Enterprise & Yes & No & No & No \\
\hline 47 & TeamLab & Yes & Yes & No & No \\
\hline 48 & AceProject & Yes & No & No & No \\
\hline 49 & Proliance & No & No & No & No \\
\hline 50 & Principal Toolbox & No & No & No & No \\
\hline 51 & FIT Issue Management & No & No & No & No \\
\hline 52 & Doolphy & Yes & No & No & No \\
\hline 53 & Deltek WelcomHome & Yes & No & No & No \\
\hline 54 & BrightWork & No & No & No & No \\
\hline 55 & VPMi & Yes & No & No & No \\
\hline 56 & Dolibarr ERP/CRM & No & Yes & No & No \\
\hline 57 & LiquidPlanner & No & No & No & No \\
\hline 58 & Smartsheet & No & No & No & No \\
\hline 59 & phpGroupWare & No & Yes & No & No \\
\hline 60 & Zoho Office Suite & Yes & No & No & No \\
\hline 61 & Cerebro & No & No & No & No \\
\hline
\end{tabular}

\begin{tabular}{|c|c|c|c|c|c|}
\hline \multicolumn{2}{|c|}{ Project Management Information System } & 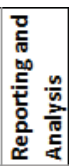 & 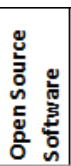 & 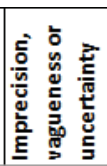 & 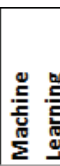 \\
\hline 62 & Gemini & No & No & No & No \\
\hline 63 & MatchWare MindView 4 & No & No & No & No \\
\hline 64 & Projectplace & No & No & No & No \\
\hline 65 & Feng Office Community Edition & No & Yes & No & No \\
\hline 66 & InLoox & Yes & No & No & No \\
\hline 67 & JIRA & Yes & No & No & No \\
\hline 68 & Traction TeamPage & No & No & No & No \\
\hline 69 & Grovesite & No & No & No & No \\
\hline 70 & LibrePlan & Yes & Yes & No & No \\
\hline 71 & Mavenlink & No & No & No & No \\
\hline 72 & DeskAway & No & No & No & No \\
\hline 73 & Bontq & No & No & No & No \\
\hline 74 & Binfire & No & No & No & No \\
\hline 75 & web2project & No & Yes & No & No \\
\hline 76 & MantisBT & No & Yes & No & No \\
\hline 77 & MicroPlanner X-Pert & No & No & No & No \\
\hline 78 & ProjectSpaces & No & No & No & No \\
\hline 79 & Teamcenter & No & No & No & No \\
\hline 80 & Ubidesk & No & No & No & No \\
\hline 81 & KForge & No & Yes & No & No \\
\hline 82 & Launchpad & No & Yes & No & No \\
\hline 83 & Wrike & No & No & No & No \\
\hline 84 & Trac & No & Yes & No & No \\
\hline 85 & Rplan & No & No & No & No \\
\hline 86 & Unawave & No & No & No & No \\
\hline 87 & Jonas Software & No & No & No & No \\
\hline 88 & Prolog Manager & No & No & No & No \\
\hline 89 & Ganttic & No & No & No & No \\
\hline 90 & Deltek Open Plan & Yes & No & No & No \\
\hline 91 & Central Desktop & No & No & No & No \\
\hline 92 & Kayako helpdesk software & No & No & No & No \\
\hline 93 & dotProject & No & Yes & No & No \\
\hline 94 & Basecamp & No & No & No & No \\
\hline 95 & codeBeamer & No & No & No & No \\
\hline 96 & Planner Suite & No & No & No & No \\
\hline 97 & TaskJuggler & No & Yes & No & No \\
\hline 98 & Collabtive & Yes & Yes & No & No \\
\hline 99 & Hall.com & No & No & No & No \\
\hline 100 & iManageProject & No & No & No & No \\
\hline 101 & KommandCore & No & No & No & No \\
\hline 102 & Financialforce.com & Yes & No & No & No \\
\hline 103 & DynaRoad & Yes & No & No & No \\
\hline 104 & OpenProj & Yes & Yes & No & No \\
\hline 105 & Huddle & No & No & No & No \\
\hline 106 & Mingle & No & No & No & No \\
\hline 107 & Hyperoffice & No & No & No & No \\
\hline 108 & GanttProject & No & Yes & No & No \\
\hline 109 & Open Workbench & No & Yes & No & No \\
\hline 110 & Microsoft Project & Yes & No & No & No \\
\hline 111 & KPlato & No & Yes & No & No \\
\hline 112 & Tom's Planner & No & No & No & No \\
\hline 113 & MindGenius & No & No & No & No \\
\hline 114 & Milestones Professional & No & No & No & No \\
\hline 115 & O3spaces & No & No & No & No \\
\hline 116 & Contactizer & No & No & No & No \\
\hline 117 & FastTrack Schedule & No & No & No & No \\
\hline 118 & LisaProject & No & No & No & No \\
\hline 119 & MacProject & No & No & No & No \\
\hline 120 & OmniPlan & No & No & No & No \\
\hline 121 & Project KickStart & No & No & No & No \\
\hline
\end{tabular}

